\title{
Retail Banking im Informationszeitalter - Trends, Geschäftsarchitektur und erste Beispiele
}

\author{
Robert Winter \\ Institut für Wirtschaftsinformatik, Universität St. Gallen \\ Müller-Friedberg-Strasse 8, 9000 St. Gallen, Schweiz \\ Robert.Winter@unisg.ch
}

\section{Einleitung}

Die Nutzung elektronischer Vertriebs- und Kommunikationskanäle (e-Commerce) oder die Entwicklung vermeintlich neuer Geschäftsmodelle (z.B. elektronische Marktplätze) sind für die Transformation in das Informationszeitalter zwar wichtig, aber nicht ausschlaggebend. Erst die zunehmende Reife einer leistungsfähigen Vernetzungs-Infrastruktur ermöglicht die radikale Neustrukturierung von Wertschöpfungsnetzwerken, die Konstruktion neuartiger, vernetzter Geschäftsmodelle und vor allem die ganzheitliche Abdeckung von Kundenbedürfnissen [Österle / Winter 2000]. Als Vernetzungs-Infrastruktur werden dabei nicht nur deren technische Komponenten wie z.B. breitbandige, kostengünstige Computernetzwerke und entsprechende Beschreibungs- und Kommunikationsstandards verstanden, sondern auch und gerade deren organisatorische Komponenten wie z.B. standardisierte elektronische Dienste oder Vertragsabwicklungsstandards.

Retail Banking ist aus verschiedenen Gründen ein ideales Anschauungsbeispiel für derartige Umstrukturierungen:

- Finanzdienstleistungen sind informatorische Leistungen, d.h. informatorische und mithin virtualisierbare Aspekte der Leistung stehen im Vordergrund, so dass Wertschöpfungsnetzwerke restrukturiert werden können, ohne auf die physischen Vernetzungsrestriktionen materieller Leistungen Rücksicht nehmen zu müssen.

- Im Finanzdienstleistungsbereich fanden aufgrund verschiedenster Regulationen lange Zeit keine radikalen Veränderungen statt. Aufgrund des sukzessiven Wegfalls dieser Regulationen werden fällige Restrukturierungen jetzt nachgeholt. 
- Im Gegensatz zu anderen Bereichen (z.B. Investment Banking) treten im Retail Banking Kundenprozesse mit hohen Wiederholungsraten auf und sind deshalb sicherer standardisierbar.

Die Wichtigkeit des letzten Punkts resultiert daraus, dass die traditionelle, das Unternehmen als Ausgangspunkt für die Leistungsdefinition nehmende Sichtweise (,inside-out“) zunehmend von einer Sichtweise abgelöst wird, die den Konsumentenprozess in den Mittelpunkt stellt und davon ausgehend Unternehmensprozesse definiert (,outside-in“). Multikanalmanagement in Form einer Entscheidung darüber, welche Produkte über welche Kanäle an welche Konsumentensegmente „vertrieben“ werden [Arnfield / Arnfield 2000], ist ein klassisches Beispiel für „,inside out“. Im Gegensatz dazu würden „outside in“ zunächst Entscheidungen darüber getroffen werden, welche Konsumentenprozesse durch Integration welcher Leistungen unterstützt werden sollen, welche Geschäftsmodelle welchen Beitrag zu dieser Integration leisten könnten und wie die Netzwerkpartner zusammenarbeiten müssen, um die Gesamtleistung effektiv und effizient zu erbringen.

Im Folgenden werden zunächst verschiedene Trends skizziert, die für Retail Banking im Informationszeitalter relevant erscheinen (Abschnitt 2). In Abschnitt 3 wird eine Geschäftsarchitektur für das Retail Banking beschrieben, die diese Trends berücksichtigt. Die Ausführungen basieren auf der in [Österle / Winter 2000] generell beschriebenen Geschäftsarchitektur des Informationszeitalters und der Adaption auf das Bankgeschäft nach [Leist / Winter 2000]. Erste Praxisbeispiele für die Implementierung ,neuer“ Rollen oder Geschäftsmodelle werden in Abschnitt 4 vorgestellt. Der Beitrag wird von einem kurzen Ausblick auf Weiterentwicklungen, Generalisierungen und Forschungsbedarfe abgeschlossen (Abschnitt 5).

\section{Trends und deren Konsequenzen}

Als Grundlage der Ableitung einer zeitgemässen Geschäftsarchitektur für Retail Banking werden im Folgenden einige Trends beschrieben. Zunächst werden generelle (d.h. branchenübergreifende, aber auch nicht originär IT-bezogene) Trends behandelt. Im zweiten Teil werden spezielle Trends im Zusammenhang mit dem Übergang ins Informationszeitalter aufgegriffen. Der dritte Teil widmet sich speziellen Trends im Finanzdienstleistungsbereich.

\subsection{Generelle Trends}

Als branchenübergreifende, nicht originär IT-bezogene Trends, die in den letzten Jahren zu weitreichenden Veränderungen von Branchen und Märkten geführt 
haben und weiter führen werden, sind insbesondere Deregulierung, Globalisierung und Individualisierung zu nennen. Deregulierung und Globalisierung erlauben Unternehmen, ihre Leistungsangebote zu redefinieren und / oder mit ihren Leistungen neue Märkte zu erschliessen. Sie zwingen ausserdem dazu, einem intensivierten Wettbewerb mit Effizienzsteigerung, Leistungsindividualisierung und / oder Fokussierung auf Kernkompetenzen zu begegnen.

Verarbeitende Branchen haben auf diese Trends folgendermassen reagiert:

- Nicht-Kernprozesse werden umfassend und im Rahmen der jeweiligen Logistik-Restriktionen global ausgelagert. Beispielsweise beschränken sich Automobilhersteller meist auf die Endmontage von Produktkomponenten, die Produktentwicklung sowie die Eigenfertigung besonders wichtiger Produktkomponenten wie z.B. von Motoren.

- Als Konsequenz aus der Konzentration auf Kernkompetenzen entstehen Wertschöpfungsnetzwerke, die sich in Form virtueller Unternehmen, Allianzen oder loser Verbünde organisieren und bestimmte Marktleistungen letztlich gemeinsam erbringen.

- Interne Geschäftsprozesse und interorganisationale Prozesse werden soweit wie möglich standardisiert. Beispielsweise erlaubt die Einführung betriebswirtschaftlicher Standardsoftware zusammen mit der Basierung auf anwendungsnahen Kommunikationsstandards wie z.B. EDIFACT, Geschäftsprozesse flexibel in einem Wertschöpfungsnetzwerk zu verteilen

- Um einerseits trotz Standardisierung von Produkten / Prozessen und globaler Präsenz ausländischer Mitbewerber „einzigartige“ Leistungen anbieten zu können und andererseits spezifische Kundenbedürfnisse befriedigen zu können, werden mehr und mehr komplexe Leistungsbündel definiert, die verschiedenste Produkte und Dienstleistungen umfassen können.

Im Retail Banking sind viele der beschriebenen Entwicklungen noch nicht in dieser Deutlichkeit eingetreten: Beschränkung auf Kernkompetenzen und konsequentes Outsourcing finden sich im vorherrschenden Universalbankkonzept genauso wenig wie standardisierte Prozesse, anwendungsnahe BranchenKommunikationsstandards (mit wenigen Ausnahmen wie z.B. SWIFT im Zahlungsverkehr) oder komplexe, individualisierbare Leistungsbündel. Vielmehr ist das Leistungsangebot vieler Retail-Banken nach wie vor produktorientiert. Abbildung 1 illustriert die Produktorientierung am Beispiel der - mittlerweile völlig umgestalteten - Retail-WebSite einer Grossbank. Die Präsentation der Produkte orientiert sich nicht etwa an Kundenprozessen, sondern lediglich am (alphabetisch sortierten) Produktnamen. Die Abbildung zeigt das Ergebnis der Suche nach Produkten, deren Name mit P beginnt. 


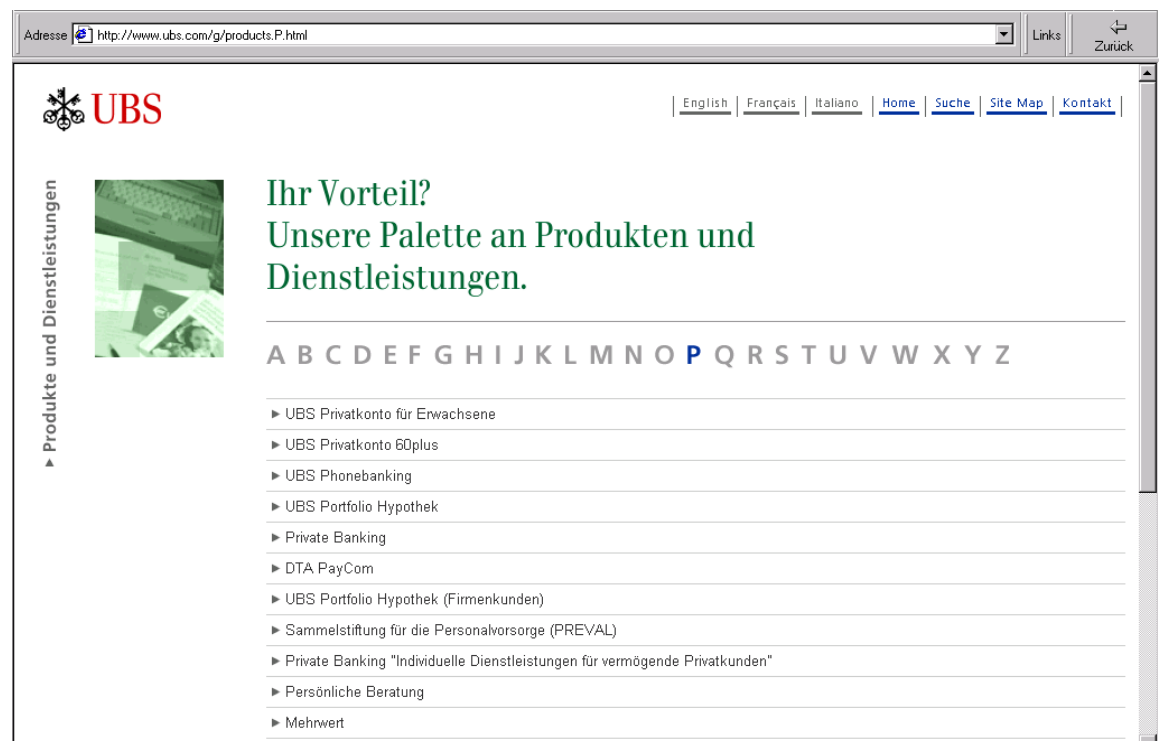

Abbildung 1. Produktorientiertes Leistungsangebot

\subsection{Spezielle Trends in Zusammenhang mit dem Übergang ins Informationszeitalter}

Innovationen der Informations- und Kommunikationstechnologie (ITInnovationen) schaffen eine technische Vernetzungsinfrastruktur, auf deren Grundlage Wertschöpfungsnetzwerke überhaupt erst realisiert werden können. Wichtige Komponenten einer solchen Vernetzungsinfrastruktur sind kostengünstig nutzbare Breitbandnetze sowie flexible und offene Beschreibungs- bzw. Kommunikationsstandards.

Ein weiterer wichtiger Enabler ist der Zugang grosser Bevölkerungskreise zum Internet. Durch die zunehmende Nutzung des Internets am Ausbildungs- und Arbeitsplatz wird es einer immer grösseren Menge von Konsumenten möglich, unmittelbar in Wertschöpfungsnetzwerke eingebunden zu werden bzw. unmittelbaren Einfluss auf die Gestaltung und / oder Bündelung von Leistungen zu nehmen [Österle / Winter 2000]. Dies geschieht einerseits durch zunehmende Nachfrage nach Leistungen, die Konsumentenprozesse ganzheitlich und nicht mehr nur bruchstückhaft unterstützen. Als Communities können Konsumenten aber auch unmittelbar in Wertschöpfungsnetzwerke eingebunden werden.

Auch die zunehmende Zahl von Zugangs- und teilweise auch Vertriebskanälen hat starken Einfluss auf die Gestaltung von Geschäftsbeziehungen. Ein immer grösserer Anteil von Konsumenten ist nicht mehr auf einen bestimmten Zugangs- bzw. 
Vertriebskanal fixiert (z.B. Filiale, Telefon), sondern verlangt einen gleichwertigen Leistungszugang über die verschiedensten Kanäle (z.B. Filiale, Fax, Telefon, e-Mail) mittels verschiedener Endgeräte (z.B. Bancomat, PC, Handy, PDA) [Cameron et al. 1999]. Für die meisten dieser Endgeräte bzw. Kanäle wird ein ortsund zeitunabhängiger Zugang nachgefragt. Bemerkenswert an dieser Entwicklung ist nicht nur die Nutzbarkeit der spezifischen Eigenschaften bestimmter Endgeräte für die Vertiefung und Ausweitung von Geschäftsbeziehungen, beispielsweise in Form wiederholter täglicher Präsenz durch SMS oder durch Bereitstellung von Mehrwertdiensten wie beispielsweise einer Terminplanung. Insbesondere die Personalisierung der über ,neue“ Kanäle und Endgeräte abgewickelten Geschäfte eröffnet eine Vielzahl von Individualisierungspotenzialen.

Für das Informationsmanagement stellen derartige IT-Innovationen erhebliche Herausforderungen dar, da sich Produktabwicklung, Kundenbeziehungsmanagement und Kanalabwicklung mehr und mehr trennen. Traditionelle Applikationen, die alle diese Funktionalitäten monolithisch implementieren, müssen deshalb aufwändig restrukturiert oder gleich in unterschiedliche, entsprechend ausgerichtete Applikationskomponenten zerlegt werden [Winter 2000].

Dienstleistungsanbieter ausserhalb des Finanzdienstleistungsbereichs (z.B. Telecoms) haben - nicht zuletzt unter dem Druck von (De-)Regulierungsbehörden IT-Potenziale zur Schaffung offener Dienstleistungsnetzwerke genutzt, in denen Netzbetreiber und Wiederverkäufer z.B. mit personalisierten Tarifen die individuellen Telekommunikationsbedürfnisse von Konsumenten immer kostengünstiger und mehr oder weniger weltweit abdecken. Konsumenten können Kommunikations-Basisleistungen verschiedenster Netzbetreiber oder Wiederverkäufer auf einfache Weise kombinieren. Selbst die aufwändige Änderung der Telefonnummer als letzte Möglichkeit, den Anbieterwechsel zu erschweren, wird bald der Vergangenheit angehören.

Im Retail Banking sind viele auch dieser Entwicklungen noch nicht eingetreten: Angebote verschiedener Banken können nur sehr selten auf einfache Weise kombiniert werden. Konsumenten-Finanzportale wie z.B. Quicken erlauben zwar die Aggregation verschiedener Kundenbeziehungen, sind aber bezüglich der unterstützten Prozesse auf Basisfunktionalitäten wie z.B. Überweisungen beschränkt. Anwendungsnahe Protokolle wie z.B. HBCI sind länderspezifisch und werden nicht von allen Banken unterstützt.

\subsection{Spezielle Trends im Finanzdienstleistungsbereich}

Die Anzahl der Konsumenten, die mehrere Bankverbindungen unterhalten, steigt seit mehreren Jahren stark [Teixeira 1999]. Nur durch das Unterhalten mehrerer Bankverbindungen ist es ja traditionell möglich, lukrative Produktangebote verschiedener Banken zu einer ganzheitlichen Problemlösung zu integrieren. Durch das Internet als Vertriebs- und Zugangskanal wird dabei nicht nur die Suche nach 
geeigneten Produkten, sondern auch der Wechsel der Bankverbindung und das Betreiben mehrerer Bankverbindungen erleichtert. Weitere Faktoren, die zu höherer Wechselbereitschaft bzw. geringerer Loyalität führen, sind die Entpersonalisierung des Retail Banking (z.B. Betreuung von Retail-Kunden durch Teams statt Einzelpersonen, zunehmende Nutzung automatisierter Zugangskanäle) sowie die erhöhte Transparenz des Produktangebots, zu dem nicht zuletzt die Wirtschaftspresse beiträgt.

„Cherry Picking“ im Sinne einer Kombination attraktiver Produkte verschiedener Anbieter durch Konsumenten wird zwar von den Banken ungern gesehen, lässt sich aber auch durch Hochhalten von Wechselkosten (z.B. Kontoauflösungsgebühren, Kundenbindungsprogramme) oder Vertrauen auf die Bequemlichkeit der Konsumenten nicht dauerhaft verhindern. Entweder muss das Produktangebot durch attraktive Produkte - im Zweifelsfall auch von Mitbewerbern - erweitert werden oder dieser Markt wird Integratoren überlassen, die dies ohnehin tun werden.

„Consumers may not use the term, but what they really want is ,aggregation“ one-stop access to all their financial data." [Berinato 2001]. Durch Aggregation von Bankverbindungen, integrierte Planungs- und Auswertungsfunktionen, ein breites Informationsangebot zu Finanzdienstleistungen verschiedener Anbieter und ggf. Community-Funktionen wie Diskussionsforen oder Produktbewertung realisieren Integratoren eine nicht unmittelbar produkt- und anbietergetriebene Unterstützung von Finanzprozessen bei Konsumenten.

Letztlich läuft auch dieser Trend auf die Trennung von Produktentwicklung, Produktabwicklung und Produktintegration im Sinne einer ganzheitlichen Prozessunterstützung hinaus [Cameron et al. 1999]. Kommen zu dieser Rollenverteilung im Finanzdienstleistungsnetzwerk noch Aushandlungs- und Abwicklungsstandards hinzu, ist die Vision „Open Finance“ weitgehend verwirklicht.

\section{Geschäftsarchitektur des Informationszeitalters für Retail Banking}

Die in Abschnitt 2 beschriebenen Trends lassen sich wie folgt zusammenfassen:

- Entstehen vernetzter Geschäftsarchitekturen: Einem durch Deregulierung und Globalisierung intensivierten Wettbewerb muss mit Effizienzsteigerung, Leistungsindividualisierung und insbesondere Fokussierung auf Kernkompetenzen begegnet werden. Durch Auslagerung von Nicht-Kernprozessen entstehen Wertschöpfungsnetzwerke, die sich in Form virtueller Unternehmen, Allianzen oder loser Verbünde organisieren und bestimmte Marktleistungen letztlich gemeinsam erbringen. Zur effektiven Implementierung der entste- 
henden interorganisationalen Prozesse müssen umfangreiche Standardisierungen vorgenommen werden.

- Mass Customization - speziell durch Bündelung: Um einerseits trotz Standardisierung von Produkten / Prozessen und globaler Präsenz ausländischer Mitbewerber „einzigartige“ Leistungen anbieten zu können und andererseits spezifische Kundenbedürfnisse befriedigen zu können, werden mehr und mehr komplexe Leistungsbündel definiert, die verschiedenste Produkte und Dienstleistungen umfassen können.

- Intensivierung der Kunden-Interaktion: Eine kostengünstige, breitbandige technische Vernetzungs-Infrastruktur zusammen mit dem Zugang grosser Bevölkerungskreise zum Internet - nicht nur privat, sondern auch und gerade am Arbeitsplatz - schaffen nicht nur grössere Transparenz und intensivieren dadurch den Wettbewerb, sondern ermöglichen es auch, grosse Kundenmengen durch Multikanalangebote und Interaktivität unmittelbar in Wertschöpfungsnetzwerke einzubinden.

- Nutzung vertieften Kundenwissens zur Individualisierung: Der hohe Anteil elektronischer Transaktionen und die Komplexität der angebotenen Leistungen erlauben eine intensivierte Sammlung von TransaktionsInformationen, die wiederum zur Individualisierung von Leistungsangeboten und aktiven Gestaltung von Kundenbeziehungen genutzt werden können.

- Open Finance: Auf Grundlage erhöhter Transparenz und geringerer Transaktionskosten werden immer häufiger Leistungskomponenten verschiedener Anbieter zu ganzheitlichen Finanzdienstleistungen kombiniert, die einen komplexen Kundenprozess ganzheitlich unterstützen. Für die Anbieter von Leistungskomponenten werden Spezialisierung, Offenheit und Performance kritische Erfolgsfaktoren. Für die „Eigentümer“ von Konsumentenbeziehungen treten dagegen ,true choice“ und „objective advice“ [Cameron et al. 1999, p.6] in den Vordergrund.

Zusammengenommen entsteht die Vision einer offenen Architektur vernetzter Geschäftsmodelle, die entweder auf die ganzheitliche Abdeckung von Konsumentenprozessen, die effiziente Abwicklung von Finanzdienstleistungen oder den Betrieb einer gemeinsamen Infrastruktur einschliesslich bestimmter Services spezialisiert sind. Auf diese Architekturvision zielen nicht nur die generellen Fokussierungs-, Vernetzungs- und Individualisierungstrends. Sie wird auch durch die IT-induzierten Standardbildungs- und Kanalunabhängigkeitstrends unterstützt und durch die starke Nachfrage nach offenen, konfigurierbaren Lösungen seitens der Konsumenten angetrieben.

Eine branchen- und prozessunabhängige Referenzarchitektur vernetzter Geschäftsmodelle wurde bereits in [Österle / Winter 2000], ihre Adaption für das Bankgeschäft erstmals in [Fugmann et al. 1999] sowie in [Leist / Winter 2000] vorgeschlagen. Im Folgenden werden als wichtigste Komponenten dieser Refe- 
renzarchitektur zunächst die verschiedenen unternehmerischen Rollen sowie die Rolle der Konsumenten beschrieben. Die Betrachtung der Interaktionen zwischen den Komponenten führt zum Gesamtmodell. Ergänzend wird die Rolle von Standards in der Geschäftsarchitektur beleuchtet und es werden grundlegende Konsequenzen der Übergangs von traditionellen zu vernetzten Geschäftsarchitekturen untersucht.

\subsection{Charakterisierung der Rollen}

In vernetzten Geschäftsarchitekturen spielt jedes Unternehmen eine bestimmte Rolle. Die meisten dieser Rollen existieren in industriellen Geschäftsarchitekturen nicht oder nur in Ansätzen [Fugmann et al. 1999; Österle / Winter 2000; Leist / Winter 2000]:

- Service Integrators integrieren (im Normalfall nicht selbsterstellte) Leistungskomponenten, um damit einen bestimmten Konsumentenprozess (z.B. die Anschaffung von Wohneigentum oder die Vermögensanlage zur Altersfinanzierung) ganzheitlich zu unterstützen. Kernkompetenz von Service Integrators ist deshalb die umfassende Kenntnis von Konsumentenbedürfnissen bzw. Konsumentenprozessen. Im Retail Banking können bankunabhängige Vertriebsorganisationen für Finanzdienstleistungen mit ausgeprägter Spezialisierung auf bestimmte Kundensegmente (z.B. MLP) als erste, noch nicht voll ausgereifte Geschäftsmodelle von Service

- Integrators angesehen werden. dene andere Service Providers oder Service Integrators. Im Gegensatz zu Service Providers decken sie keine Bedürfnisse ganzheitlich ab, sondern spezialisieren sich auf die effiziente Herstellung spezifischer Leistungskomponenten in grossen Mengen. Im Retail Banking können Transaktionsbanken, Clearinghäuser, Brokerage-Unternehmen und andere, „fabrikähnliche“ Geschäftsmodelle als Shared Service Provider angesehen werden.

- Exclusive Service Providers erzeugen ebenfalls Leistungskomponenten für andere Service Providers oder Service Integrators. Im Gegensatz zu Shared Service Providers werden aber nicht grosse Mengen von Leistungskomponenten für mehrere Abnehmer produziert, sondern spezifischere Leistungskomponenten für einen oder wenige Abnehmer und damit in eher kleineren Mengen. An die Stelle von Kunden- bzw. Kundenprozesskenntnis (Service Integrator) bzw. Kostenführerschaft (Shared Service Provider) treten als HauptErfolgsfaktor Schnelligkeit, Flexibilität und / oder spezifische Prozesskompetenzen. Im Retail Banking können Aktivitäten wie z.B. Risikomanagement oder Produktentwicklung als Exclusive Services angesehen werden. Allerdings ist es bisher nicht üblich, um solche Aktivitäten herum eigene Geschäftsmodelle aufzubauen. 
- Public Services stellen Leistungskomponenten bereit, die keinen spezifischen bankfachlichen Charakter haben, aber zur Abwicklung der Leistungserstellung unverzichtbar sind. Im engeren Sinne handelt es sich dabei um Dienste mit quasi-hoheitlicher Funktion wie z.B. Zertifizierung oder Authentifizierung. Im weiteren Sinne können auch andere elektronische Dienste wie z.B. Informationsdienste, Applikationsbetrieb, Katalogdienste, Zahlungsabwicklung und Logistikabwicklung darunter subsumiert werden. Im Retail Banking finden sich bereits verschiedenste solcher Dienste, sei es im quasihoheitlichen Bereich in Form halbstaatlicher Zertifizierungsstellen oder im allgemeinen Bereich in Form von Outsourcern, ASP's oder Auskunftsdiensten.

\subsection{Rolle der Konsumenten}

Das traditionelle „Vertriebs“-Verständnis basiert auf der Segmentierung von Konsumenten entsprechend organisatorischer Strukturen, demographischer Merkmale, historischer Absatzdaten und / oder Absatzprognosen. Auf Grundlage der Konsumentensegmentierung, des Produktprogramms und der Potenziale der verschiedenen Vertriebskanäle wird dann geplant, welche Produkte bzw. Produktlinien über welche Kanäle zu welchen Konditionen an welche Konsumentensegmente „vertrieben" werden sollen. Natürlich wird auch in dieser Situation versucht, Konsumentenbedürfnisse durch Lieferung nutzenstiftender Produkte über präferierte Kanäle zu attraktiven Konditionen zu befriedigen. Die industrielle - d.h. an Produktionsprozessen orientierte - Strukturierung der Geschäftsmodelle verhindert jedoch meist, dass komplexe Konsumentenprozesse ganzheitlich unterstützt werden können. Es bleibt letztlich den Konsumenten überlassen, verschiedene Produkte möglicherweise unterschiedlicher Hersteller zu kombinieren.

In einer vernetzten Geschäftsarchitektur werden die Geschäftsmodelle der Service Integrators um Konsumentenprozesse, die ein ausreichendes Profitabilitätspotenzial versprechen, herum entworfen. Dabei wird davon ausgegangen, dass Konsumenten eine ganzheitliche, spezifische Unterstützung insbesondere von komplexen Prozessen durch Service Integrators dem „Selbst-Kombinieren“ vorziehen.

Der bereits angesprochene Trend „vom Produkt zur Problemlösung“ (z.B. im Touristikbereich) unterstützt diese Vermutung, während der Trend zur Individualisierung ihr entgegenzustehen scheint. Moderne IT-Applikationen wie z.B. individualisierbare Portale oder 1:1-Marketing ermöglichen es jedoch, dass sich Service Integrators nicht auf pauschale Unterstützungsangebote für grosse Kundensegmente beschränken müssen, sondern automatisiert Angebote bereitstellen können, die jedem/r Konsumenten/in individuell erscheinen (,Mass Customization“).

Es muss jedoch davon ausgegangen werden, dass ein bestimmter Anteil von Konsumenten und Konsumentenprozessen durch vorstrukturierte, ganzheitliche Pro- 
zessunterstützung nicht erreicht werden kann (z.B. für sehr spezifische Konsumentenprozesse oder-segmente) oder will.

\subsection{Gesamtmodell}

Das Gesamtmodell beschreibt nicht die einzelnen Komponenten des Wertschöpfungsnetzwerks, sondern vielmehr deren Zusammenwirken.

Ausgangspunkt sind die Konsumentenprozesse, hinter denen in den meisten Fällen auch spezifische Kundensegmente stehen. Jeweils bestimmte Konsumentenprozesse werden durch Service Integrators ganzheitlich unterstützt, wobei ein Konsumentenprozess (bzw. Kundensegment) auch durch verschiedene Service Integrators unterstützt werden kann. Bei Längsschnittbetrachtungen ist dies allgemein der Fall, da sich die Unterstützung oft auf Konsumentenprozesse bezieht, die nur selten oder gar einmalig auftreten: Das Kundensegment „wandert“ dann vom einen prozessspezifischen Service Integrator zum nächsten.

Service Integrators sowie Service Providers beziehen Leistungskomponenten von Shared Service Providers, Exclusive Service Providers und Public Services. Dabei werden im Normalfall mehrstufige Leistungsketten entstehen. Während Exclusive Service Providers nur einen oder wenige Service Providers bzw. Service Integrators beliefern und deshalb u.U. aus Effizienzgründen direkte Vernetzungen aufbauen, erfolgen alle anderen Vernetzungen im Normalfall über eine gemeinsame, offene Kollaborationsinfrastruktur, den sog. Business Bus. An die Stelle einer Vielzahl bilateraler Vernetzungen zwischen Geschäftsmodellen tritt dann jeweils nur ein einziger „Adapter“ zum Business Bus.

Der direkte Zugriff von Konsumenten auf Leistungskomponenten von Service Providers ist - sinnvollerweise über den Business Bus - möglich, wenn seitens der Konsumenten die Nutzung eines Service Integrators nicht erwünscht ist oder wenn die zu unterstützenden Prozesse so spezifisch sind, dass eine individuelle Kombination von Leistungskomponenten unausweichlich ist.

Das Gesamtmodell des Zusammenwirkens der verschiedenen Rollen wird als „Geschäftsarchitektur des Informationszeitalters“ durch Abbildung 2 llustriert. 


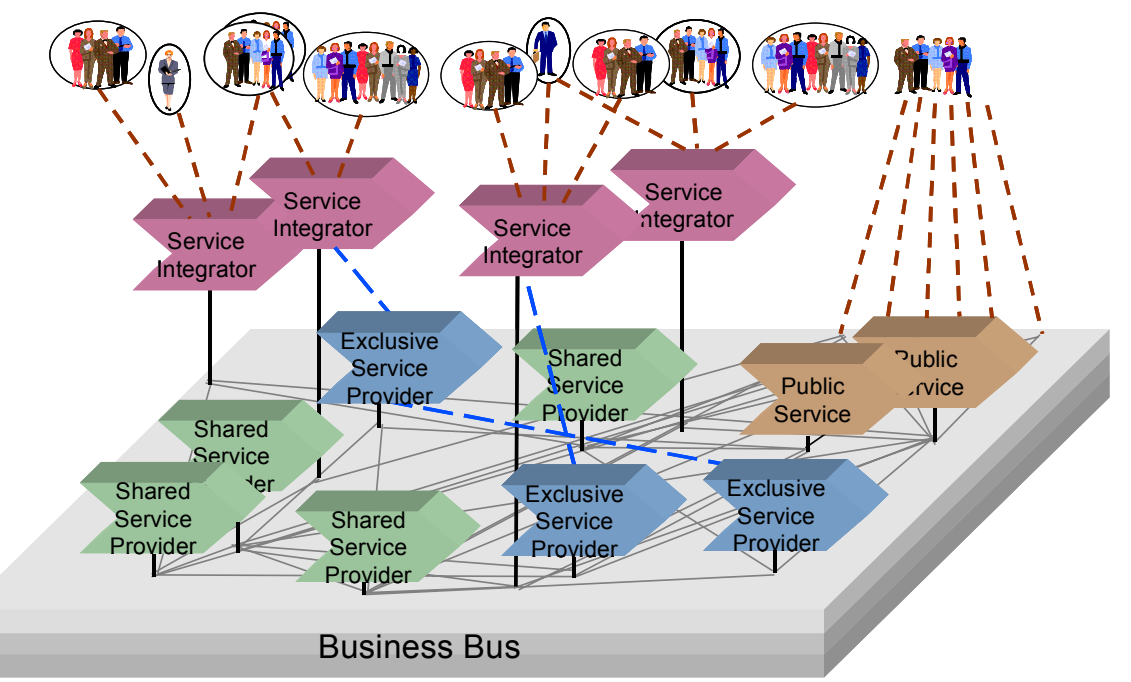

Abbildung 2. Geschäftsarchitektur des Informationszeitalters

\subsection{Standards}

Vernetzte Geschäftsarchitekturen können nur effizient realisiert werden (und nur dann einen Fortschritt gegenüber traditionellen Geschäftsarchitekturen darstellen), wenn eine Vielzahl von Standards auf Geschäfts-, Prozess- und Applikationsebene (zur Abgrenzung der Architekturebenen vgl. den Beitrag von Leist in diesem Band) sowie entsprechende Softwarepakete zur Verfügung stehen.

Während die Notwendigkeit von Kommunikationsstandards zur Systemvernetzung schon früh erkannt wurde und eine Vielzahl entsprechender Standards weit verbreitet ist (z.B. Ethernet, TCP/IP, HTTP, XML), entwickeln sich anwendungsnahe Applikations- und Prozessstandards (z.B. EDIFACT, HBCI) oder gar Geschäftsabwicklungsstandards (z.B. für Produktkataloge, für die Preisvereinbarung oder für Service Level Agreements) erst langsam. Aber gerade Standards auf Geschäfts- und Prozessebene sind für die Schaffung offener Infrastrukturen, in denen Geschäftsbeziehungen flexibel eingegangen, gesteuert und beendet werden müssen, enorm wichtig (vgl. den Beitrag von Hugentobler in diesem Band) 


\subsection{Konsequenzen des Übergangs von traditionellen zu vernetzten Geschäftsarchitekturen}

Als wesentliche Unterschiede zwischen der Geschäftsarchitektur des Informationszeitalters und traditionellen, industriellen Geschäftsarchitekturen sind festzuhalten:

1. Während sich in traditionellen Geschäftsarchitekturen alle Rollen an Produktionsprozessen ausrichten, stehen bei der Definition des Geschäftsmodells von Service Integrators nicht Produktions-, sondern Konsumentenprozesse im Mittelpunkt.

2. Während in traditionellen Geschäftsarchitekturen individuelle Vernetzungen zwischen Geschäftspartnern dominieren, bedarf die Geschäftsarchitektur des Informationszeitalters einer offenen, von fast allen Geschäftsmodellen genutzten Infrastruktur, dem Business Bus.

Industrielle Geschäftsarchitekturen sind oft durch alle Rollen abdeckende Geschäftsmodell-Konglomerate sowie durch - wenn überhaupt - lokale Vernetzungs-Infrastrukturen gekennzeichnet. Im traditionellen Retail Banking bildete sich diese Struktur im deutschen Sprachraum in Form der Koexistenz einer mittleren Zahl von Universalbanken sowie einiger Konzern- bzw. Verbundplattformen. In der Geschäftsarchitektur des Informationszeitalters finden sich dagegen relativ wenige, dafür aber sehr grosse Shared Service Providers, viele kleine, spezialisierte Exclusive Service Providers und sehr viele, spezifisch auf Konsumentenprozesse und / oder -segmente zugeschnittene Service Integrators sowie eine umfassende Vernetzungs-Infrastruktur. Die Auswirkungen des Übergangs zum Informationszeitalter liegen damit auf der Hand: Geschäftsmodell-Konglomerate (z.B. in Form von Universalbanken) zerfallen in Netzwerke, wobei die Zahl der „Fabriken“ dramatisch abnehmen muss, im Gegensatz dazu aber konsumentenprozessorientierte Geschäftsmodelle in grosser Zahl etabliert werden müssen. Zur Veranschaulichung der (zahlenmässigen) Entwicklung von einer industriellen Geschäftsarchitektur (links) hin zu einer Geschäftsarchitektur des Informationszeitalters (rechts) dient Abbildung 3. 


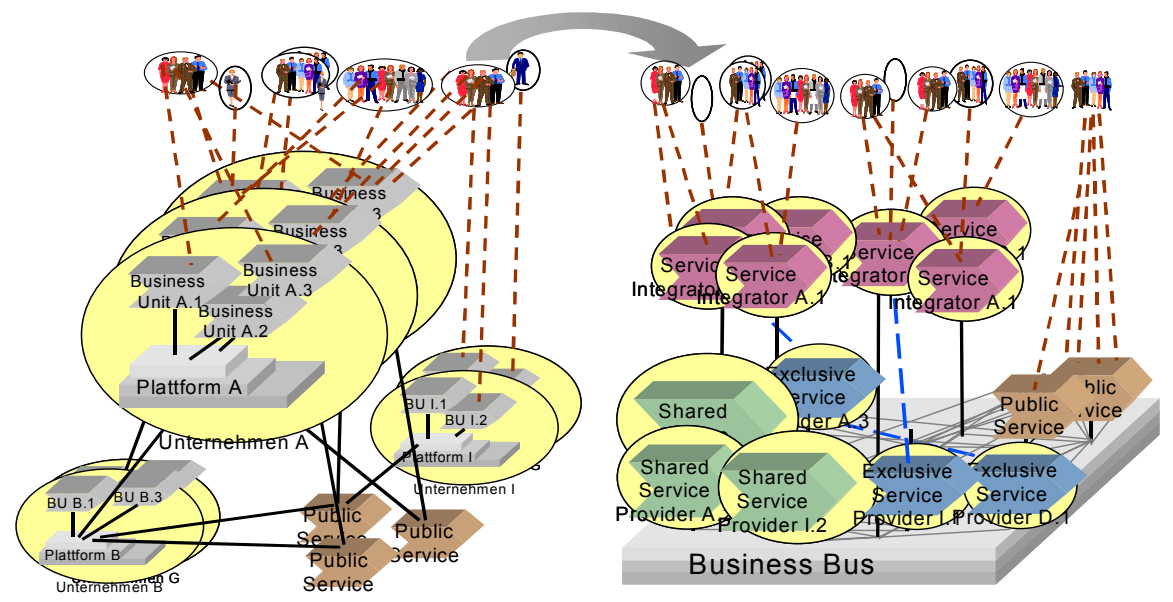

Abbildung 3. Wirkungen des Übergangs in das Informationszeitalter

\section{Erste Beispiele für Retail Banking im Informationszeitalter}

Das in Abschnitt 3 vorgestellte Modell der Geschäftsarchitektur des Informationszeitalters ist eine Vision, die hilfreich ist, um Entwicklungslinien zu erkennen, das aktuell erreichte Stadium der Transformation einordnen zu können und Gestaltungsprioritäten abzuleiten. Da der Übergang von industriellen Geschäftsarchitekturen zu Geschäftsarchitekturen des Informationszeitalters massive Transformationen mit sich bringt (z.B. Ausbildung von Netzwerkfähigkeit, Implementierung neuer Geschäftsmodelle, u.U. umfassende Redefinition des Leistungsprogramms), befinden sich vernetzte Geschäftsarchitekturen noch im Entwicklungsstadium insbesondere in einer auch in traditionellen Strukturen ökonomisch attraktiven Branche wie dem Retail Banking. Anhand verschiedener Beispiele aus dem Retail Banking und mit ihm zusammenwachsenden Bereichen wird im Folgenden gezeigt, wo und wie die Transformation stattfindet.

\subsection{Vernetzte Geschäftsarchitekturen}

Vernetzte Geschäftsarchitekturen sind ein Phänomen, das nicht erst in der jüngsten Vergangenheit auftritt. Verbundunternehmen wie z.B. die Volks- und Raiffeisenbanken oder die Sparkassen des deutschen Sprachraums haben schon früh ausgewählte Geschäftsprozesse an zentrale Einheiten ausgelagert und so Shared 
Service Provider geschaffen. So nimmt z.B. eine durchschnittliche Volks- oder Raiffeisenbank Zahlungsverkehrs- und Kontoführungsdienstleistungen eines Verbandsrechenzentrums, Clearing-, Handels- und Depotverwaltungsfunktionen einer Verbands-Zentralbank sowie Produktabwicklungen einer Verbands-Bausparkasse, einer Verbands-Versicherung und eines Verbands-Leasingunternehmens wahr. Beratung und Rechnungsprüfung erfolgen ebenfalls im Normalfall durch verbandseigene Dienstleistungsunternehmen. Bezogen auf das Grundmodell der Geschäftsarchitektur des Informationszeitalters könnte dann z.B. die Kreditbearbeitung als Exclusive Service Provider und die Bündelung aller Leistungskomponenten sowie die Kundenbetreuung als Service Integrator angesehen werden. In Abbitung 4 wird Aufgabenteilung im Volks- und Raiffeisenverbund als Geseträftsarchitektur des Informationszeitalters dargestellt.

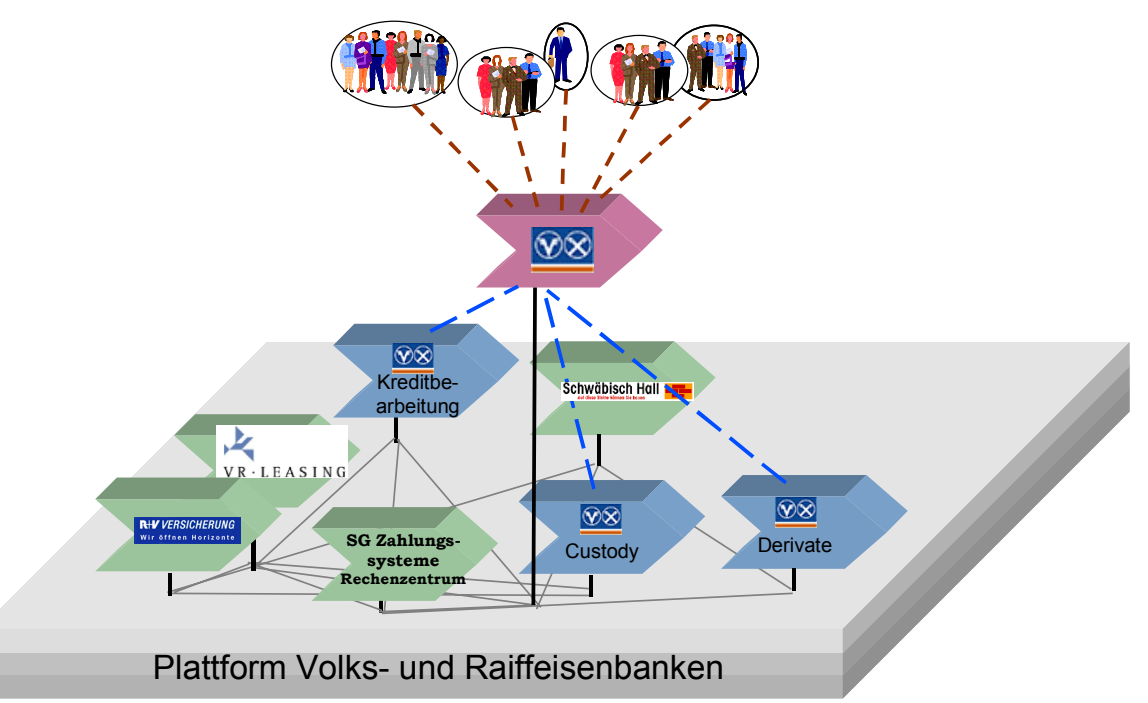

\section{Abbildung 4. Vernetzung bei deutschen Volks- und Raiffeisenbanken}

Verschiedene Formen vernetzter Geschäftsarchitekturen finden sich auch in virtuellen Banken (siehe folgender Gliederungspunkt) und in Unternehmensnetzwerken, die durch Zerlegung z.B. einer Universalbank entstanden sind. Allerdings sind virtuelle Unternehmen und Konzernverbünde weit weniger als Geschäftsnetzwerke anzusehen als z.B. Verbands- oder Genossenschaftsstrukturen, weil das entsprechende Netzwerk mehr oder weniger zentral gesteuert wird, das Netzwerk nicht vollständig offen ist und / oder die Netzwerkunternehmen durch Besitz- und Beteiligungsstrukturen aneinander gebunden sind. In allen Fällen basiert die Vernetzung zudem nicht auf einer umfassenden Infrastruktur, sondern auf mehr oder weniger „lokalen“ Plattformen.

Die in den letzten Jahren beobachtbare Zerlegung monolithischer Universalbanken mit dem Ziel, lose und zumindest teilweise offene Unternehmensnetzwerke zu 
bilden, entspricht zwar im Grundsatz den hier beschriebenen Transformationen zur Geschäftsarchitektur des Informationszeitalters. Allerdings zeigt die Unsicherheit beim Zuschnitt der Netzwerkknoten (auf Zerlegungen folgen häufig Reintegrationen, siehe z.B. die Bildung von „Nicht-Investmentbank-Bereichen“ bei Deutscher Bank und Credit Suisse), dass sich Referenz-Vernetzungen erst langsam herausbilden.

\subsection{Service Integrators}

Grundlage des Geschäftsmodells von Service Integrators ist ein Konsumentenprozess oder eine Gruppe verwandter Konsumentenprozesse. Für die Strukturierung der angebotenen Unterstützungsleistungen gibt es verschiedene Ansätze. So können als Ansatzpunkt bestimmte (Teil-)Prozesse oder bestimmte Ereignisse differenziert werden. Für beide Ansätze gibt es viele Beispiele:

- Als (Teil-)Prozesse im Retail Banking unterscheidet z.B. die Commerzbank „everyday life“, „realizing plans“ (d.h. grössere Anschaffungen oder Reisen), "securing the future“ und „wealth and independence“ (d.h. Geldanlage) [Commerzbank 2000].

- Als Ereignisse, die Konsumenten zu Entscheidungen im Finanzdienstleistungsbereich veranlassen, unterscheidet z.B. Quicken Autokauf, Heirat, Hauskauf, Geburt, Wechsel der Arbeitsstelle, Scheidung / Trennung und Übergang in den Ruhestand [Quicken 1999].

(Teil-)Prozesse und Ereignisse im Finanzdienstleistungsbereich sind oft eng miteinander verknüpft, so dass sich meist Mischformen finden. So unterscheidet z.B. First Direct zwischen „enjoying success“, „travelling“, „,separating“, „improving your home“, „having a family“, „buying a home“, „retiring“, „getting married“, „changing jobs“, „,buying a car“" und „changing banks“ [First Direct 1999].

Für die Differenzierung zwischen traditionellen Vertriebsorganisationen und Service Integrators ist jedoch nicht die Art der Leistungsstrukturierung ausschlaggebend, sondern das Ausmass der Loslösung vom Produktionsprozess. Dieses kann durch zwei Kriterien operationalisiert werden:

1. Werden Leistungskomponenten gebündelt oder werden Leistungen so angeboten, wie sie produziert werden?

2. Ist die Trennung zwischen Produktionsprozessen und Konsumentenprozessunterstützung transparent, z.B. durch Unterscheidung von (produktionsprozessbezogenen) Produktmarken und der Marke des Service Integrator?

Auf der Grundlage dieser Kriterien lassen sich drei Erscheinungsformen von Vertriebsorganisationen unterscheiden, die das Konzept des Service Integrator unterschiedlich stark realisieren: 
- Virtuelle Bank (weder Bündelung noch Markentransparenz): Eine „virtuelle Bank" bietet Leistungskomponenten verschiedenster Service Provider unter eigener (Bank-)Marke an, ohne dass für den Endkonsumenten transparent wird, wer die Leistungskomponenten erstellt. Beispielsweise vertreibt die Advance Bank Produkte unter eigener Marke, obwohl sie durch Service Provider abgewickelt werden. Da letztlich nur eine gemeinsame Marke für fremdbezogene Produkte entwickelt wird, unterscheidet sich dieses Geschäftsmodell nur unwesentlich von traditionellen Retail Banking-Angeboten.

- Infomediary (keine Bündelung, aber Markentransparenz): Leistungskomponenten verschiedenster Service Provider werden durch den „Infomediary“ unter der Marke des jeweiligen Produzenten angeboten (z.B. Quicken). Der Mehrwert des Infomediary besteht aus dem Angebot von „true choice“ und „objective advice“ [Cameron et al. 1999, p.6], wobei letzteres insbesondere durch umfangreiche Informationsangebote im Umfeld der Leistungskomponenten erreicht wird. Die Abwicklung der Produkte erfolgt transparent, d.h. durch die jeweiligen Service Provider. Zwar werden in diesem Geschäftsmodell Produkte durch Informationen ergänzt, stehen aber weiterhin im Mittelpunkt, so dass es nicht als „reiner“ Service Provider bezeichnet werden kann.

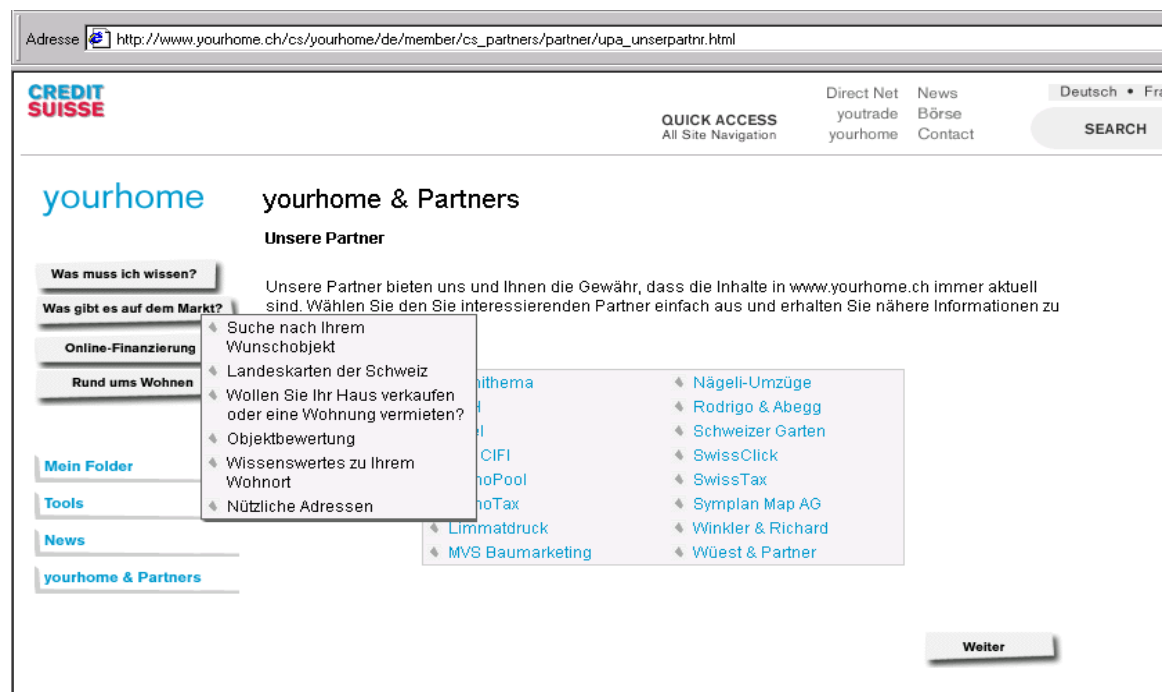

Abbildung 5. Endkonsumenten-Prozessportal yourhome [Yourhome 2000]

- Service Integrator i.e.S. (sowohl Bündelung wie auch Markentransparenz): Leistungskomponenten verschiedenster Service Provider werden zur Unterstützung eines oder weniger Konsumentenprozesse integriert, wobei die Marken der kombinierten Produkte im Normalfall erhalten bleiben (d.h. die Integration für den Endkonsumenten transparent ist) und allenfalls durch die Mar- 
ke des Integrators ergänzt werden. Der Service Integrator gewährleistet nicht nur die Auswahl geeigneter Service Provider, sondern wickelt zumindest einige Leistungen auch selbst ab. Abjturg - illustriert dieses Geschäftsmodell am Beispiel von yourhome [ourtome 2000]. Dieses EndkonsumentenProzessportal integriert verschiedenste Finanzdienstleistungen, Informationsdienste, Hilfsmittel (z.B. Entwurfs-, Lokalisierungs- und Bewertungswerkzeuge) und Marktübersichten rund um den Prozess „Wohneigentumserwerb“ in der Schweiz. Erst wenn - z.B. aufgrund der umfassenden Unterstützung der unterstützte Prozess gegenüber den Leistungskomponenten in den Vordergrund tritt, wird das Geschäftsmodell des Service Integrators in Reinform umgesetzt [Baumöl / Winter 2001]. Im Beispiel yourhome wird diese Umsetzung jedoch solange unvollständig sein, wie sie von einem einzigen Finanzdienstleistungsanbieter dominiert wird und damit weder „true choice“ noch „objective advice“ gewährleisten kann.

Service Integrators sind im Normalfall branchenübergreifend ausgerichtet. So ist z.B. in yourhome nur eines der vielen Partnerunternehmen eine Bank, weil Finanzierungsprozesse nur einen Teilausschnitt des Wohneigentumerwerbsprozesses darstellen - auch wenn diese Bank das gesamte Portal finanziert und damit dominiert. Während für Service Provider traditionelle Branchenstrukturen nach wie vor z.B. für die Definition von Referenzprozessen relevant sind, wird sich für Service Integrators eine alternative „Branchen“-Strukturierung herausbilden, die sich statt an Produktionsprozessen an Konsumentenprozessen orientiert [Winter 2001].

\subsection{Service Providers}

Grundlage des Geschäftsmodells von Service Providers sind Produktionsprozesse, für die im Fall von Shared Service Providers Kostenführerschaft, im Fall von Exclusive Service Providers dagegen besondere Flexibilität, besondere Schnelligkeit oder spezifische Prozesskompetenzen entwickelt werden können. Während sich für Kostenführerschaft die Analogie der Massenfertigung aufdrängt, können für Kernkompetenz-basierte Produktionsprozesse als Analogien in traditionellen Branchen z.B. Engineering, Design oder individualisierte Kleinserien- bzw. Einzelfertigung herangezogen werden.

Fabrik-ähnliche Geschäftsmodelle finden sich zur Zeit im Retail Banking insbesondere in Form der sog. Transaktionsbanken, die mehr und mehr nicht nur verbands- oder konzernweite Leistungskomponenten (z.B. Zahlungsverkehr, Kontoführung, Wertschriftenverwahrung) herstellen, sondern auch für Kunden ausserhalb des Verbands bzw. des Konzerns zur Verfügung stehen sollen. So konnte z.B. die European Transaction Bank e.t.b. AG, eine Tochter der Deutschen Bank, die deutschen Sparda-Banken als Mandant gewinnen [European Transaction Bank 2001]. Die tatsächliche Ausgestaltung eines solchen Insourcing erweist sich in der Praxis aber oft als äusserst komplex und kostspielig, so dass die Nachhaltigkeit 
derartiger Geschäftsmodelle noch nicht abschliessend beurteilt werden kann. Neben Transaktionsbanken finden sich - insbesondere in den USA - bereits verschiedenste „E-Services“, die als Service Provider angesehen werden können: Billpoint (www.billpoint.com), E-Loan (www.e-loan.com), EmoneyMail (www.emoneymail.com), Checkfree (www.checkfree.com) und Fiserv (www.fiserv.com) sind nur einige der standardisierten Dienstleistungsprovider, die Zahlungsverkehr, Bill Presentment, Kontoführung u.a. als elektronische Dienste anbieten [Net Worth 2001]. Auch Application Service Providing, d.h. der mandantenfähige Betrieb von Applikationen für Retail-Banken, kann als Service Providing im Sinne der hier vorgestellten Definition angesehen werden. So betreibt z.B. die Schweizerische Real-Time Center AG ein mandantenfähiges Bankensystem für verschiedene Kantonalbanken, Regionalbanken, Privatbanken und die Migrosbank [Real-Time Center 2001]. Es darf erwartet werden, dass die sukzessive Überwindung politischer und kultureller „Disabler“ der Standardisierung, zunehmende Erfahrung bei der Standardisierung von Geschäftsprozessen und Geschäftsmodellen sowie nicht zuletzt zunehmender Kostendruck den attraktivsten dieser Geschäftsmodelle ausreichend grosse Märkte eröffnen, um profitabel umgesetzt werden zu können.

Engineering-, Design- bzw. Kleinserienfertigungs-ähnliche Geschäftsmodelle finden sich zur Zeit im Retail Banking z.B. in Form von Produktspezialisten, die innovative Produkte (z.B. im Fondsbereich) schnell situativ entwickeln und abwickeln können. Aufgrund der zunehmenden Markttransparenz finden attraktive Produkte mehr und mehr Eingang in das Angebot nicht nur eines, sondern einer Vielzahl von Service Integrators. So finden sich z.B. im Fondsangebot der Credit Suisse Produkte von Pictet, Goldmann Sachs, HSBC, Clariden, Fidelity, ABN Amro u.a. [Credit Suisse 2001]; Im Angebot der Schweizerischen Postbank finden sich Fondsprodukte der Credit Suisse Asset Management [Postbank 2001].

\subsection{Business Bus}

Die Schaffung applikationsnaher, verbands- und konzernübergreifender Standards muss als äusserst schwierig angesehen werden, da Standardisierungsbemühungen nicht nur mit syntaktischen und semantischen Anpassungsproblemen der Nomenklaturen und Geschäftsprozesse der betroffenen Institute, sondern auch und gerade mit Macht- und Kulturproblemen belastet sind. Diese Problematik stellt wohl das Haupthindernis für die schnelle Transformation zur Geschäftsarchitektur des Informationszeitalters im Retail Banking da; Ihre Auswirkungen sind z.B. in der Schweiz am Scheitern ambitiöser Projekte wie der Schaffung einer gemeinsamen Transaktionsbank, der Einrichtung einer gemeinsamen Zertifizierungsstelle oder der Schaffung einer gemeinsamen Plattform für Bill Presentment spürbar (siehe z.B. [Swisskey 2001]). Sobald - wie z.B. innerhalb von Verbänden, Konzernen oder Allianzen - politische und kulturelle Probleme in den Hintergrund treten, zeigen Beispiele wie die aktuelle Weiterentwicklung von HBCI oder die Schaf- 
fung einer „European Value Chain“ [Strebel 2001], dass applikationsnahe Standardisierung durchaus erfolgversprechend betrieben werden kann.

\section{Ausblick}

Dieser Beitrag basiert auf der Analyse verschiedener Trends, die zu einem Architekturmodell zusammengeführt werden, für dessen Validierung einige erste Umsetzungsbeispiele herangezogen werden. An verschiedenen Stellen wurde erwähnt, dass die konsequente Realisierung der Vision z.B. an politischen (Macht-)Bedingungen, kulturellen Differenzen (z.B. zwischen traditionell völlig unterschiedlich entwickelten Branchen), nationalen Regulationen, fehlender Erfahrung mit Prozess- und Geschäftsmodellstandardisierung oder fehlender Flexibilität der verfügbaren IT-Plattformen scheitern kann. Darüber hinaus ist jede Transformation grundsätzlichen Gefahren wie z.B. dem zu frühen Angehen „richtiger“ Innovationen (z.B. Bill Presentment, Prozessportale), „falschen“ Innovationen (z.B. ineffiziente Verteilung von Geschäftsprozessen im Netzwerk) oder zu langem Abwarten ausgesetzt. Diese Erkenntnisse relativieren die Radikalität der in diesem Beitrag beschriebenen Veränderungen. Nichtsdestoweniger waren sich auf dem Gründungsworkshop des Kompetenzzentrums „Bankenarchitekturen des Informationszeitalters“ Anfang 1999 alle Bankenvertreter einig, dass die seinerzeitigen Geschäftsmodelle zwischen 2001 und 2003 radikale Veränderungen durchmachen würden.

Als wesentliche Herausforderungen für die strategische Planung im Retail Banking erscheinen die folgenden Problemfelder:

- Identifikation der Rolle(n) der eigenen Unternehmung in der Geschäftsarchitektur, die sie aufgrund der eigenen Kompetenzen und der Kompetenzen der Mitbewerber effektiv und effizient ausfüllen kann.

- Redefinition von Kundenbeziehungen und Kundenorientierung entsprechend der als sinnvoll identifizierten Rolle(n) in der Geschäftsarchitektur. Service Integrators haben vollkommen andere Kunden und pflegen vollkommen andere Kundenbeziehungen als Service Providers.

- Erkennen von Netzwerkfähigkeit als notwendige Kernkompetenz für alle Rollen in der Geschäftsarchitektur und Umsetzen entsprechender Massnahmen - insbesondere Offenheit und Standardisierung.

- Schaffung eines Instrumentariums, das es erlaubt, Strategien, Geschäftsmodelle, Geschäftsprozesse und Applikationssysteme transparent und arbeitsteilig zu entwickeln, zu dokumentieren und zu kommunizieren. Nur wenn auf allen Ebenen entsprechende Spezifikationen vorliegen und kommuniziert wer- 
den können, besteht die Chance, Standards zu entwickeln und Netzwerkpartner auszutauschen.

Die Ergebnisse des Kompetenzzentrums „Bankenarchitekturen des Informationszeitalters", die in diesem Band dokumentiert sind, leisten dazu einen wichtigen Beitrag.

\section{Literatur}

[Arnfield / Arnfield 2000]

Arnfield, R.; Arnfield, B.: Multi-channel Financial Services: Strategies for Optimising the Delivery Channel Mix, Lafferty Publications, December 2000

[Baumöl / Winter 2001]

Baumöl, U.; Winter, R.: Intentions Value Networks - A Business Model of the Information Age, in: Miranda P.; Sharp, B.; Pakstas, A.; Filipe, J. (eds.): ICEIS 2001 - Proc. of the Third International Conference on Enterprise Information Systems, vol. 2, Setubal: ICEIS Press 2001, pp. $1075-1080$

[Berinato 2001]

Berinato, S.: Building a better bank, in: The Standard, February - March 2001, pp. 82-86, http://www.thestandard.com/grok, March 2001

[Cameron 1999]

Cameron, B.; Mines, C.; Hermsdorf, L.: New IT for open finance, The Forrester Report, Juli 1999.

[Commerzbank 2000]

Services for private customers, http://www.commerzbank.com/navigate/ priv_frm.htm, Dezember 2000

[Credit Suisse 2001]

Aktienfonds - unsere Empfehlung, https://entry.credit-suisse.ch/cs/leo/ p/s/de/produkte/anlagefonds/anf_aktien_3.html, September 2001

[European Transaction Bank 2001]

European Transaction Bank, http://www.etb-ag.com/deutsch/index.html, September 2001

[First Direct 1999] 
First Direct Home, http://www.firstdirect.com/services/frame.html, September 1999

[Fugmann et al. 1999]

Fugmann T.; Heinrich, B.; Leist, S.; Winter, R.: Banking im Informationszeitalter - Formen und Gestaltungsfragen von Wertschöpfungsnetzwerken im Bankbereich, in: Steiner, M.; Dittmar T.; Willinsky, C. (Hrsg.): Elektronische Dienstleistungswirtschaft und Financial Engineering, Münster: Schüling 1999, S. 237-261

[Langlinais / deLeon 1999]

Langlinais, T.; deLeon, A.: Winning the e-economy with "Intention Value Networks", in: EIU Strategic Finance, 1999, The Economist Intelligence Unit Ltd., pp. 50-68

[Leist / Winter 2000]

Leist, S.; Winter, R.: Finanzdienstleistungen im Informationszeitalter Vision, Referenzmodell und Transformation, in: Belz, C.; Bieger, T. (Hrsg.): Dienstleistungskompetenz und innovative Geschäftsmodelle, St. Gallen: Thexis 2000, S. 150-166

[Net Worth 2001]

Looking for Net Worth, in: The Standard, February - March 2001, pp. 42-45, http://www.thestandard.com/grok, March 2001

[Österle / Winter 2000]

Österle, H.; Winter, R.: Business Engineering, in: Österle, H.; Winter, R. (Hrsg.): Business Engineering - Auf dem Weg zum Unternehmen des Informationszeitalters, Berlin etc.: Springer 2000, S. 3-20

[Postbank 2001]

Postbank Immobilienfonds, http://www.postbank.de/cgi-bin/WebObjects/ PBWebPage.woa/3/wo/mu2567CR6iCN3169BW32UYLYU4Y/12.0.17.

1.1.CPBTopicDocumentMain.11.1.0.CPBComponentDoc.0.

PBFondsPopup.1, September 2001

[Quicken 1999]

InsureMarket Life Events Advisor, http://www.insuremarket.com/event/ noframes/kids.htm, September 1999

[Real-Time Center 2001]

Real-Time Center AG, http://www.rtc.ch/, September 2001

[Swisskey 2001] 
Swisskey stellt Ausgabe von digitalen Zertifikaten ein, http://www.swisskey.ch/prod/webpage?in_lang=1\&in_node=7, Mai 2001

[Strebel 2001]

Strebel, B.: Maintenance wird Kerngeschäft, Schweizer Bank, 7/2001, S. 14-15

[Teixeira 1999]

Teixeira, D.: Internet Financial Services: Customer in Control, Presentation at the ABA E-Customer Forum, July 1999

[Winter 2000]

Winter, R.: Zur Positionierung und Weiterentwicklung des Data Warehousing in der betrieblichen Applikationsarchitektur, in: Schmidt, H. (Hrsg.): Modellierung betrieblicher Informationssysteme (Proc. der MobIS-Fachtagung 2000), Rundbrief der GI-Fachgruppe 5.10, Bd. 7 (2000), Nr. 1, S. 23-38

[Winter 2001]

Winter, R.: Mass Customization and Beyond - Evolution of Customer Centricity in Financial Services, to appear in: Rautenstrauch, C.; Turowski, K.; Seelmann-Eggebert, R. (eds.): Mass Customization in Progress - Information Systems and Management Principals, Berlin etc.: Springer 2001

[Yourhome 2000]

Yourhome \& Partners, http://www.yourhome.ch/cs/yourhome/de/ member/cs_partners/partner/upa_unserpartnr.html, Dezember 2000 